\title{
Publisher Correction: An adaptive supramolecular hydrogel comprising self-sorting double nanofibre networks
}

Hajime Shigemitsu, Takahiro Fujisaku, Wataru Tanaka, Ryou Kubota, Saori Minami, Kenji Urayama and Itaru Hamachi

Correction to: Nature Nanotechnology https://doi.org/10.1038/s41565-017-0026-6, published online 8 January 2018.

In the version of this Article originally published online, in Fig. 4b, in the lower-right image, the value of $r$ was incorrect; it should have read ' $r=0.72$ '. This has now been corrected in all versions of the Article.

Published online: 29 January 2018

https://doi.org/10.1038/s41565-018-0065-7 